\title{
Bacterial Imprinting at Pickering Emulsion Interfaces
}

\author{
Xiantao Shen, ${ }^{[a]}$ Johan Svensson Bonde, ${ }^{[a]}$ Tripta Kamra, ${ }^{[a]}$ Leif Bülow, ${ }^{[a]}$ Jack C. Leo, ${ }^{[b]}$ Dirk Linke, ${ }^{[b]}$ and \\ Lei $Y e^{*[a]}$
}

\begin{abstract}
We demonstrate that the tendency of bacteria to assemble at oil-water interface can be utilized to create microbial recognition sites on the surface of polymer beads. In this work, two different groups of bacteria were first treated with acryloylfunctionalized chitosan, and then used to stabilize an oil-in-water emulsion composed of crosslinking monomer dispersed in aqueous buffer. Polymerization of the oil phase followed by removal of the bacterial template resulted in well-defined polymer beads bearing bacterial imprints. Chemical passivation of chitosan and cell displacement assays indicate that the bacterial recognition on the polymer beads was dependent on the nature of the pre-polymer and the target bacteria. The functional materials for microbial recognition show great potential for constructing cell-cell communication networks, biosensors, and new platforms for testing antibiotic drugs.
\end{abstract}

Research at the intersection between microbiology and nano/micro fabrication represents an enormous challenge. ${ }^{[1]}$ Within this context, controlled self-assembly of microorganisms is a subject of substantial interest in many biomedical and bacteriological studies (e.g. cell-cell communication and cellsurface interaction). ${ }^{[1 b]}$ In the literature, controlled bacterial assembly has been achieved mainly by soft lithography patterning and through cell-wall engineering. ${ }^{[2]}$ These strategies exploit the chemical interactions between bacteria and surfaces to control microbial adhesion. The existing methods to pattern bacteria-binding surfaces are incapable of offering high selectivity, therefore it is necessary to develop new synthetic strategies that can offer specific bacterial recognition surfaces.

One way to achieving selective bacterial recognition surfaces is via the molecular imprinting technique.$^{[3]}$ Molecular imprinting allows the preparation of synthetic polymers (molecularly imprinted polymers, MIPs) with specific binding sites for predetermined target molecules. ${ }^{[4]}$ Because of their high specificity, MIPs have found many practical applications spanning affinity separation, catalysis and chemical sensing.$^{[5]}$ However, when imprinting large templates (e.g. proteins, viruses and bacteria), some non-trivial problems are encountered. ${ }^{[c, d]}$ The first problem is that, as living organisms, bacteria are not rigid and it is therefore difficult to generate well-defined cavities during an imprinting reaction. The second problem is that traditional MIPs are highly cross-linked, making it difficult for bacteria to reach the binding sites buried in the interior of the MIPs. To overcome these difficulties, several new approaches including the

[a] Dr. X, Shen, Dr. J. S. Bonde, T. Kamra, Prof. L. Bülow, Dr. L. Ye Division of Pure and Applied Biochemistry

Lund University

Box 124, 22100 Lund, Sweden

E-mail: Lei.Ye@tbiokem.Ith.se

[b] Dr. J. C. Leo, Dr. D. Linke

Department of Biosciences

University of Oslo

P.O.Box 1066 Blindern, 0316 Oslo, Norway

Supporting information for this article is given via a link at the end of the document. "stamping" method, ${ }^{[6]}$ sol-gel chemistry,${ }^{[7]}$ colloidal imprints, ${ }^{[8]}$ and the lithographic process ${ }^{[9]}$ have been widely used to create bacterial imprints on the polymer matrix.

In this work, we developed a novel method to synthesize bacterial recognition polymers by exploiting the capability of bacteria to self-assemble at an oil-water interface. By selecting pre-polymers (e.g. carbohydrate) which show high affinity for the target bacteria, we demonstrate that bacterial recognition polymers can be prepared from emulsions stabilized by bacteriapre-polymer networks. In the literature, particle stabilized emulsion is also named Pickering emulsion. ${ }^{[10]}$ The basic principle of droplet stabilization is the partitioning of solid particles between the two immiscible liquids. ${ }^{[10 a]}$ Since it was first reported, Pickering emulsion has been exploited in various applications, such as oil recovery, cosmetic preparations, waste water treatment, and fabrication of functional materials with complex architectures. ${ }^{[10 b]}$ For example, using Pickering emulsion polymerization, MIPs capable of recognizing small organic molecules and proteins have been synthesized in our laboratory. [11 ] Besides amphiphilic solid particles, living microorganisms have also been used as particle stabilizers to prepare Pickering emulsions. ${ }^{[12]}$ In this context, the self-assembly of a bacteria-chitosan network at the oil-water interface was found sufficient to stabilize the Pickering emulsion.

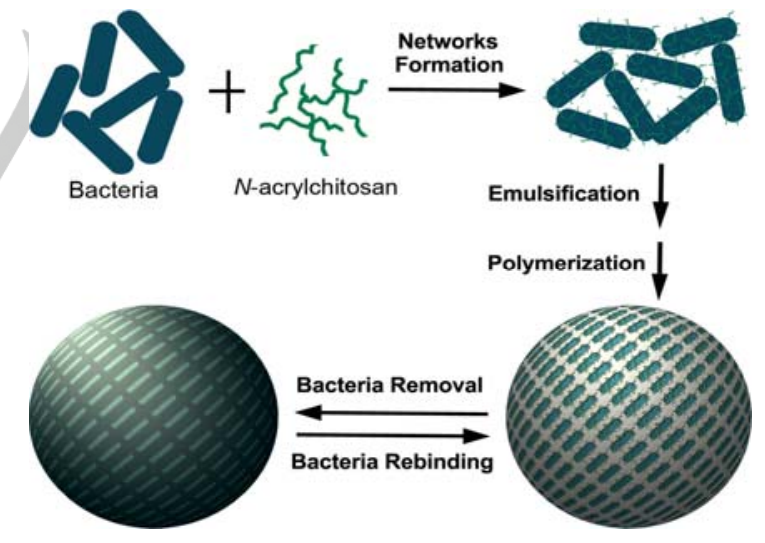

Scheme 1. Interfacial bacteria imprinting by Pickering emulsion polymerization.

Our synthetic approach for preparing Bacteria-Imprinted Polymer (BIP) by Pickering emulsion polymerization is schematically shown in Scheme 1. First, negatively charged bacteria are assembled with a positively charged vinylcontaining pre-polymer, and the obtained bacteria-pre-polymer complex is then used as particle stabilizer to construct a stable emulsion of a cross-linking monomer (the oil phase) in water. The oil phase is polymerized by means of free radical initiation, which also causes the pre-polymer to be covalently fixed on the core of the polymer beads. In the last step, the bacterial template is removed from the polymer beads, leaving behind bacteria-imprinted sites on the bead surface. The advantage of this method resides on its general applicability, as a large variety 
of pre-polymers can be selected based on their specific molecular interactions with the target bacteria.

Here, the vinyl-containing pre-polymer, $N$-acrylchitosan (NAC) was synthesized by reacting acryloyl chloride with the amino groups of the glucosamine units in chitosan (Figure S1). The fluorescence study in Figure S5 indicated that approximately $16 \%$ of the amino groups on chitosan were acrylated. A low degree of acrylation was used deliberately to make sure that the obtained NAC has enough free amino groups to bind the bacteria through electrostatic interactions. ${ }^{[12 a]}$ The pendant acryloyl groups in the modified chitosan can still allow NAC to be co-polymerized with the cross-linkers in the oil phase.
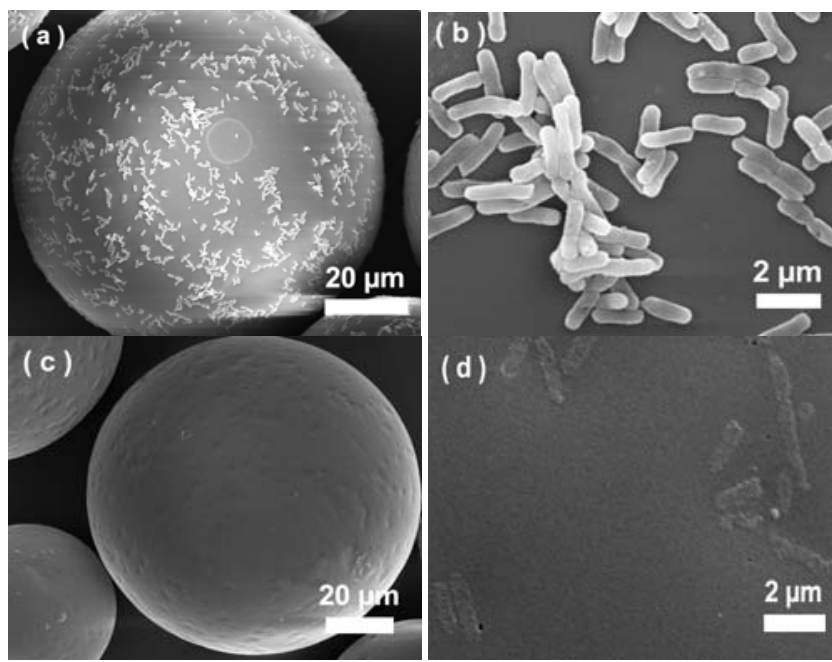

Figure 1. SEM images of bacteria imprinted polymer beads before $(a, b)$ and after $(\mathrm{c}, \mathrm{d})$ removal of the $E$. coli template.

During the preparation of Pickering emulsion, the oil phase was composed of cross-linkers and initiator. After mixing the oil phase with an aqueous suspension of the bacteria-NAC complex, a stable emulsion was obtained by shaking the mixture vigorously (Figure S6c). It should be noted that no stable Pickering emulsion could be obtained if the bacteria or NAC alone was used (Figure S6), indicating that the bacteria-NAC complex was essential for stabilizing the Pickering emulsion (Figure 1). This result is in agreement with the literature, ${ }^{[12 a]}$ which suggested that the bacteria-chitosan network irreversibly cover the oil-water interface in the Pickering emulsion.

The stable Pickering emulsion was polymerized at room temperature. After removal of the bacteria template by solvent extraction, BIP beads were obtained. The BIP beads were named E-BIP and M-BIP when rod-shaped Escherichia coli cells (E. coli) and spherical Micrococcus luteus (M. luteus) were used as the bacterial templates, respectively. Fluorescence microscope images in Figure S9 indicate that the particle size of the BIP beads is $110 \pm 17 \mu \mathrm{m}$. The surface morphology of the BIP beads before and after bacterial removal was studied by scanning electron microscopy (SEM). Before solvent extraction, the E-BIP beads prepared in PBS buffer containing the template bacteria $\left(\mathrm{OD}_{600}=2, \sim 5 \times 10^{8} \mathrm{cfu} \mathrm{\textrm {mL } ^ { - 1 }}\right)$ had clearly visible $E$. coli cells on their surface (Figure $1 a-b$ and Figure S7). After the bacteria were removed, tailor made cavities were left on the surface of the E-BIP beads, which are easily accessible for bacterial binding (Figure 1c-d). The recognition performance of the bacteria on the BIP beads was first studied by using the following bacterial species as representatives: $E$. coli, Lactobacillus sp. (LB790), M. luteus and Enterococcus faecium (E. faecium) (Table 1) ${ }^{[13]}$ Figure 2a-b show the adsorption of $E$. coli and $M$. luteus by $E$-BIP and M-BIP beads. We found that both $E$-BIP and M-BIP beads showed preferential binding of the corresponding template cells, indicating that BIP beads possessed high selectivity between rod-shaped and spherical bacteria. Interestingly, this selectivity between the rod-shaped bacteria and the spherical bacteria was stronger than the selectivity between the cells within the same group (see Figure $2 c-d)$. The selective bacterial binding results suggest that the present bacterial imprinting at Pickering emulsion interfaces is an effective way to produce specific bacterial recognition surfaces. However, the microbial imprinting has not reached the same precision as that can be achieved by imprinting small molecules.
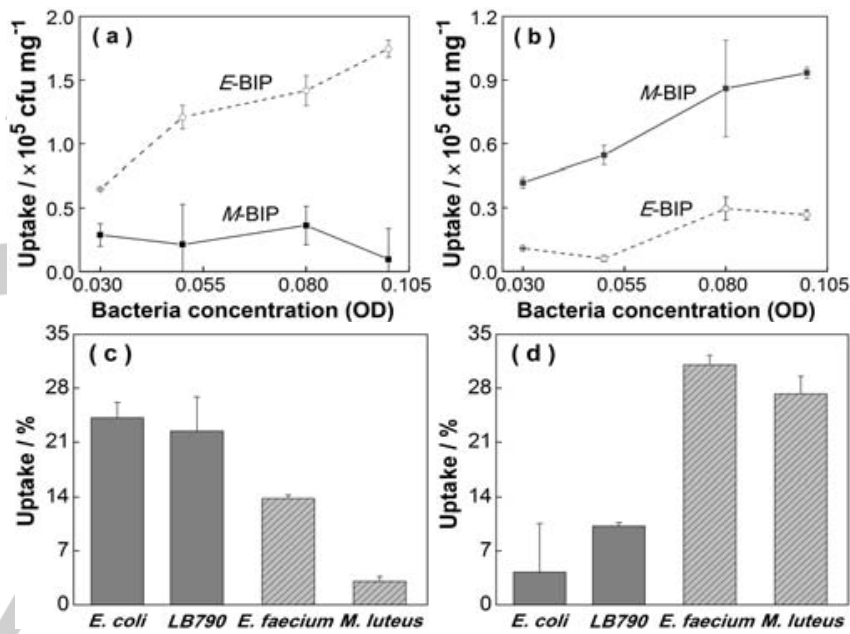

Figure 2. Uptake of $E$. coli (a) and M. luteus (b) cells with different concentrations by E-BIP and M-BIP beads. Uptake of different cells $\left(\mathrm{OD}_{600}=0.05\right)$ by $E$-BIP (c) and M-BIP (d) beads. Conditions: $50 \mathrm{mg}$ of polymer beads in $2 \mathrm{~mL}$ of PBS buffer ( $\mathrm{pH} 7.2)$, incubated with bacteria at $4{ }^{\circ} \mathrm{C}$ for $3 \mathrm{~h}$.

To confirm the importance of the pre-polymers for bacterial binding, we passivated the amino groups on BIP beads via acylation reaction with three different acid anhydrides. The $E$ BIP beads treated with acetic anhydride, maleic anhydride and trifluoroacetic anhydride were named RP-A, RP-M and RP-T, respectively. Uptake of $E$. coli expressing green fluorescent protein (GFP-E. coli) by different polymers was then studied. In Figure $3 a$, the E-BIP beads displayed higher $E$. coli binding than the reference polymer beads, suggesting that the electrostatic interaction between the cationic NAC on the polymer beads and the negatively charged $E$. coli surface play an important role for bacterial binding. The negatively charged RP-M beads (bearing carboxyl groups on surface) showed almost no binding for $E$. coli cells, indicating that the negatively charged surface effectively repelled the $E$. coli cells. Compared to the RP-M beads, the reference polymer beads covered by alkyl (RP-A) and fluoroalkyl 
groups (RP-T) showed higher E. coli binding, presumably mediated by hydrophobic interactions.
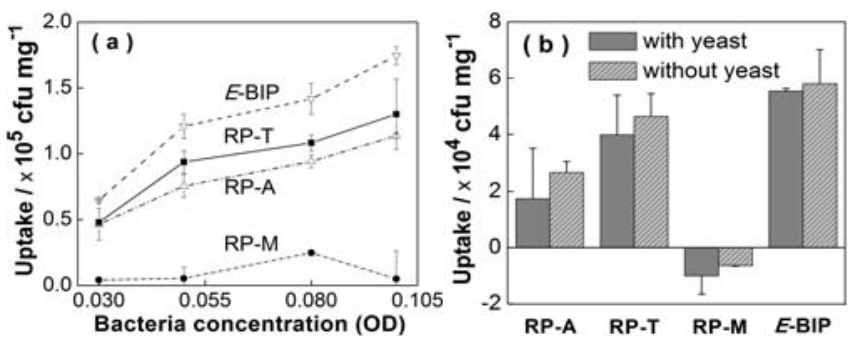

Figure 3. (a) Uptake of GFP-E. coli cells measured at different initial concentrations. Conditions: $50 \mathrm{mg}$ of polymer beads in $2 \mathrm{~mL}$ of PBS buffer ( $\mathrm{pH}$ 7.2), incubated with GFP-E. coli at $4{ }^{\circ} \mathrm{C}$ for $3 \mathrm{~h}$. (b) Uptake of GFP-E. coli cells $\left(\mathrm{OD}_{600}=0.03\right)$ in the presence of yeast cells $\left(\mathrm{OD}_{600}=0.24\right)$. Conditions: $50 \mathrm{mg}$ of polymer beads in $1 \mathrm{~mL}$ of PBS buffer $(\mathrm{pH} 7.2)$, incubated at $4{ }^{\circ} \mathrm{C}$ for $3 \mathrm{~h}$.

The selectivity of bacteria binding on BIP beads was further investigated by measuring uptake of GFP-E. coli cells in the presence of excess Saccharomyces cerevisiae yeast cells. As shown in Figure $3 \mathrm{~b}$, addition of the yeast cells had almost no influence on the uptake of the target $E$. coli cells by the different polymer beads. This result can be explained as the effect of the microbial size: the much larger yeast cells do not fit into $E$. coli imprinted sites. Despite the strong negative charge on surface (with a $\zeta$ potential of $-39.3 \mathrm{mV}$ measured in water ${ }^{[13 \mathrm{a}]}$ ) and the larger size, the yeast cells were unable to compete with $E$. coli for the imprinted sites. ${ }^{[14]}$ Therefore, besides the electrostatic interaction, the bacterial size also affects the bacterial binding.

Table 1. Displacement $(D P)$ of bound mCherry-E. coli from E-BIP beads with different competing bacteria.

\begin{tabular}{|c|c|c|c|c|c|}
\hline Competitor & E. coli & B. $s p$. & LB790 & E. faecium & M. Iuteus \\
\hline$\zeta$-Potential ${ }^{*}$ & $-20 m V^{[12 a]}$ & $-15 m V^{[13 b]}$ & $-5 \mathrm{mV}^{[13 c]}$ & $-30 m V^{[13 d]}$ & $-10 m V^{[13 e]}$ \\
\hline Shape & Rod & Rod & Rod & Sphere & Sphere \\
\hline Size $(\mu \mathrm{m})$ & $\begin{array}{l}\phi 0.4-0.8 \\
\times 2.0\end{array}$ & $\begin{array}{r}\phi 0.6-0.9 \\
\times 1.5-4.0\end{array}$ & $\begin{array}{r}\phi 0.7-1.1 \\
\times 2.0-4.2\end{array}$ & $\sim \phi 0.8$ & $\sim \phi 0.8$ \\
\hline$D P^{* *}$ & $63.0 \pm 4.0$ & $38.3 \pm 8.5$ & $37.1 \pm 3.9$ & $30.4 \pm 5.4$ & $7.7 \pm 0.9$ \\
\hline
\end{tabular}

${ }^{*}$ At $\mathrm{pH} \sim 7 ;^{* *}$ Displacement $(D P)$ of mCherry-E. coli is calculated as $D P(\%)=$ $\left[\left(\right.\right.$ bound $_{0}-{\left.\text { bound }) / \text { bound }_{0}\right] \times 100 \text {, where bound }}_{0}$ and bound are the amount of mCherry- $E$. coli cells $\left(\mathrm{OD}_{600}=0.03\right)$ bound by $E$-BIP beads in the absence and presence of the competing bacteria $\left(\mathrm{OD}_{600}=0.24\right)$, respectively. Conditions: 50 $\mathrm{mg}$ of beads in $1 \mathrm{~mL}$ of PBS buffer $(\mathrm{pH} 7.2)$, incubated at $4{ }^{\circ} \mathrm{C}$ for $3 \mathrm{~h}$.

To confirm the importance of the chemical recognition and the physical size on bacteria binding, competitive bacterial binding experiments were carried out. Here, E. coli expressing mCherry (mCherry-E. coli) was selected instead of GFP-E. coli, because $\mathrm{mCherry}-E$. coli show longer emission wavelength $(612 \mathrm{~nm})$ and lower background interference. During the binding, an excess of various bacteria were added to a mixture of E-BIP beads and $m$ Cherry-E. coli $\left(\mathrm{OD}_{600}=0.05\right)$ in PBS. Depending on the shape and surface charge similarity, the added bacteria were expected to displace the mCherry- $E$. coli from the $E$-BIP beads to different degrees. Table 1 shows that mCherry- $E$. coli was most efficiently displaced by $E$. coli (without mCherry) $>\angle B 790=$ Bacillus sphaericus (B. sp.) $>$ E. faecium $>M$. luteus. This order of efficiency can be explained by comparing the electrostatic nature and the size between mCherry- $E$. coli and the competing bacteria. For example, the $E$. coli cells (without mCherry) have the same shape and surface charge as mCherry- $E$. coli, hence $E$. coli cells efficiently displaced the mCherry-E. coli from the $E$ BIP beads. M. luteus, which are spherical with a negative charge surface, showed only a low effect on the binding of mCherry- $E$. coli. Interestingly, LB790 (with a higher $\zeta$-potential and a larger size) and $B$. sp. (with a lower $\zeta$-potential and a smaller size) showed the same degree of displacement. Moreover, compared to $M$. luteus, E. faecium (which are similar in size to M. luteus but differ in $\zeta$-potential) showed a higher degree of $E$. coli displacement, which agrees with results presented in Figure $2 \mathrm{c}$ and $2 \mathrm{~d}$. These results confirm that the bacterial binding by the BIP beads is affected by both the nature of the pre-polymers and the size of the target bacteria.

Figure 1a shows that $E$. coli cells self-assembled into aggregates during the Pickering emulsion polymerization, suggesting that the bacteria-imprinted surface may be used to deplete the microorganism more efficiently. To confirm this hypothesis, we incubated E-BIP and RP-M beads with a high concentration of $E$. coli in both PBS buffer and water, and carried out SEM analysis for the polymer beads. In PBS, a high density of $E$. coli was observed on the E-BIP beads (Figure 4a), whereas RP-M showed a much lower bacterial density on the surface (Figure S12d). In water, high E. coli binding to the E-BIP surface was also observed (Figure 4b, Figure S13a-b). Again, no obvious self-assembly of cells could be observed on the reference polymer (Figure S12, Figure S13). Based on these results, we suggest that the bacteria-imprinted sites on the $E$ BIP surface facilitates the initial $E$. coli binding, and the surface bound $E$. coli could attract more bacterial cells through a selfassembly process. Obviously, the possibility of using imprinted surface to control cellular self-assembly can have practical applications, e.g. in studying cell differentiation, preventing pathogen spreading, and tissue engineering.

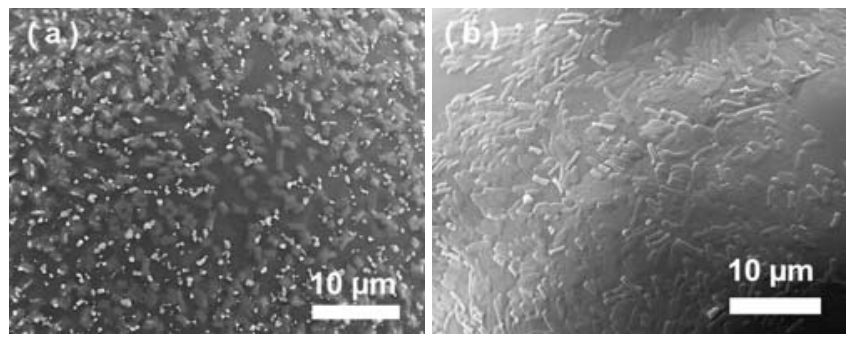

Figure 4. SEM images of bacteria self-assembly on E-BIP beads in PBS buffer (a) and in water (b). In image a, the white crystals are from PBS buffer. Conditions: $5 \mathrm{mg}$ of polymer beads incubated in $1 \mathrm{~mL}$ of $E$. coli suspension $\left(\mathrm{OD}_{600}=0.5\right)$ at $4{ }^{\circ} \mathrm{C}$ for $24 \mathrm{~h}$.

In conclusion, using bacteria as particle stabilizer and at the same time as microbial template, we prepared BIP beads using Pickering emulsion polymerization. During the imprinting, the pre-polymers that matched the target cell surface could be 
preferentially selected. This synthetic strategy is versatile and can be extended to a large variety of biological supramolecular systems as long as they can be surface activated by complexion with suitable functional pre-polymers. The imprinting reaction is straightforward and can be scaled up. The binding experiments of the two groups of bacteria provide strong evidence that the bacterial recognition on the BIP beads is dependent on the nature of the pre-polymers and the target bacteria. Interestingly, the bacteria-imprinted sites could facilitate the self-assembly of the target bacteria under overloading conditions. For biological applications, similar cellular imprinted surfaces may be utilized to control cell-cell communications and differentiation. The fine structure of the imprinted surface may also provide special microenvironments to improve cell viability, and thus lead to many new exciting possibilities in the life science area.

\section{Acknowledgements}

This work was supported by the Swedish Research Council (VR), the Swedish Research Council for Environment, Agricultural Sciences and Spatial Planning (FORMAS), and VISTA, a basic research program funded by Statoil in collaboration with the Norwegian Academy of Science and Letters.

Keywords: Bacterial recognition $\cdot$ molecular imprinting $\bullet$ prepolymer $\cdot$ Pickering emulsion $\cdot$ cellular self-assembly

[1] a) D. B. Weibel, W. R. Diluzio, G. M. Whitesides, Nat. Rev. Microbiol 2007, 5, 209. b) Y. Hiratsuka, M. Miyata, T. Tada, T. Uyeda, Proc. Natl. Acad. Sci. U.S.A. 2006, 103, 13618.

[2] a) A. Cerf, J.-C. Cau, C. Vieu, Colloids Surf. B: Biointerfaces 2008, 65, 285. b) J. A. Prescher, C. R. Bertozzi, Nat. Chem. Biol. 2005, 1, 13.

[3] a) H. Jeon, G. Kim, Langmuir 2012, 28, 13423. b) C. Alexander, E. N. Vulfson, Adv. Mater. 1997, 9, 751. c) A. Aherne, C. Alexander, M. J. Payne, N. Perez, E. N. Vulfson, J. Am. Chem. Soc. 1996, 118, 8771.

[4] a) J. L. Urraca, C. S. A. Aureliano, E. Schillinger, H. Esselmann, J. Wiltfang, B. Sellergren, J. Am. Chem. Soc. 2011, 133, 9220. b) A Cutivet, C. Schembri, J. Kovensky, K. Haupt, J. Am. Chem. Soc. 2009 131, 14699. c) K. Takeda, A. Kuwahara, K. Ohmori, T. Takeuchi, J. Am. Chem. Soc. 2009, 131, 8833. d) G. Pan, Y. Zhang, Y. Ma, C. Li, H. Zhang, Angew. Chem. Int. Ed. 2011, 50, 11731; Angew. Chem. 2011 123, 11935. e) R. Simon, M. E. Collins, D. A. Spivak, Anal. Chim. Acta 2007, 591, 7 .

[5] a) Y. Hoshino, H. Koide, T. Urakami, H. Kanazawa, T. Kodama, N. Oku, K. J. Shea, J. Am. Chem. Soc. 2010, 132, 6644. b) X. Shen, L. Zhu, N. Wang, L. Ye, H. Tang, Chem. Commun. 2012, 48, 788. c) H. Shi, W.-B. Tsai, M. D. Garrison, S. Ferrari, B. D. Ratner, Nature 1999, 398, 593. d) R. Schirhagl, P. A. Lieberzeit, F. Dickert, Adv. Mater. 2010, 22, 2078.

[6] a) K. Ren, R. N. Zare, ACS Nano 2012, 6, 4314. b) K. Ren, N. Banaei, R. N. Zare, ACS Nano 2013, 7, 6031.

[7] a) T. Cohen, J. Starosvetsky, U. Cheruti, R. Armon, Int. J. Mol. Sci. 2010, 11, 1236. b) J. Starosvetsky, T. Cohen, U. Cheruti, D. Dragoljub, R. Armon, J. Biomater. Nanobiotechnol. 2012, 3, 499.

[8] a) J. Borovička, W. J. Metheringham, L. A. Madden, C. D. Walton, S. D. Stoyanov, V. N. Paunov, J. Am. Chem. Soc. 2013, 135, 5282. b) J. Borovička, S. D. Stoyanov, V. N. Paunov, Nanoscale 2013, 5, 8560.

[9] a) S. D. Harvey, G. M. Mong, R. M. Ozanich, J. S. Mclean, S. M. Goodwin, N. B. Valentine, J. K. Fredrickson, Anal. Bioanal. Chem. 2006, 386, 211. b) T. Nagaoka, H. Shiigi, S. Tokonami, K. Saimatsu, J. Flow Injection Anal. 2012, 29, 7 .
[10] a) G. M. Whitesides, B. Grzybowski, Science 2002, 295, 2418. b) Z. Wang, M. C. M. van Oers, F. P. J. T. Rutjes, J. C. M. van Hest, Angew. Chem. Int. Ed. 2012, 51, 10746; Angew. Chem. 2012, 124, 10904. c) S J. Crossley, Faria, M. Shen, D. E. Resasco, Science 2010, 327, 68.

[11] a) X. Shen, L. Ye, Chem. Commun. 2011, 47, 10359. b) X. Shen, L. Ye, Macromolecules 2011, 44, 5631. c) X. Shen, C. Xu, L. Ye, Soft Matter 2012, 8, 3169. d) X. Shen, T. Zhou, L. Ye, Chem. Commun. 2012, 48, 8198.

[ 12 ] a) P. Wongkongkatep, K. Manopwisedjaroen, P. Tiposoth, S. Archakunakorn, T. Pongtharangkul, M. Suphantharika, K. Honda, I. Hamachi, J. Wongkongkatep, Langmuir 2012, 28, 5729. b) J. K. Ferri, P. Carl, N. Gorevski, T. P. Russell, Q. Wang, A. Böker, A. Fery, Soft Matter 2008, 4, 2259. c) J. T. Russell, Y. Lin, A. Böker, L. Su, P. Carl, H. Zettl, J. $\mathrm{He}, \mathrm{K}$. Sill, R. Tangirala, T. Emrick, K. Littrell, P. Thiyagarajan, D. Cookson, A. Fery, Q. Wang, T. P. Russell, Angew. Chem., Int. Ed. 2005, 44, 2420; Angew. Chem. 2005, 117, 2472.

[13] a) Ph. Thonart, M. Custinnet, M. Paquot, Enzyme Microb. Technol. 1982, 4, 191. b) A. Kongpol, T. Pongtharangkul, J. Honda, Kato, K. H. Ohtake, A. S. Vangnai, FEMS Microbiol. Lett. 2009, 297, 225. c) L. HernándezRodríguez, C. Lobato-Calleros, D.J. Pimentel-González, E.J. VernonCarter, Food Hydrocolloids 2014, 36, 181. d) J. J. Johanson, L. Feriancikova, S. Xu, Environ. Sci. Technol. 2012, 46, 1511. e) S. S. Khan, A. Mukherjee, N. Chandrasekaran, Colloids Surf. B 2011, 87,129. 